\title{
Japanese encephalitis virus infects porcine kidney epithelial PK15 cells via clathrin- and cholesterol- dependent endocytosis
}

\author{
Songbai Yang, Minhui He, Xiangdong Liu, Xinyun Li, Bin Fan ${ }^{*}$ and Shuhong Zhao*
}

\begin{abstract}
Background: Japanese encephalitis virus (JEV) is a mosquito-borne flavivirus that causes acute viral encephalitis in humans. Pigs are important amplifiers of JEV. The entry mechanism of JEV into porcine cells remains largely unknown. In this study, we present a study of the internalization mechanism of JEV in porcine kidney epithelial PK15 cells.

Results: We demonstrated that the disruption of the lipid raft by cholesterol depletion with methyl- $\beta$-cyclodextrin $(M \beta C D)$ reduced JEV infection. We also found that the knockdown of clathrin by small interfering RNA (siRNA) significantly reduced JEV-infected cells and JEV E-glycoprotein levels, suggesting that JEV utilizes clathrin-dependent endocytosis. In contrast, the knockdown of caveolin-1, a principal component of caveolae, had only a small (although statistically significant) effect on JEV infection, however, JEV entry was not affected by genistein. These results suggested that JEV entry was independent of caveolae.
\end{abstract}

Conclusions: Taken together, our results demonstrate that JEV enters porcine kidney epithelial PK15 cells through cholesterol- and clathrin-mediated endocytosis.

Keywords: JEV, PK15, Cholesterol, Caveolin-1, Clathrin, Infection

\section{Background}

Japanese encephalitis virus (JEV) is a mosquito-borne flavivirus that belongs to the family Flaviviridae. JEV is one of the most important endemic encephalitides and can cause acute viral encephalitis, of which there are approximately 50,000 cases in humans annually [1]. JEV can infect a wide range of cells of different origins. Pigs act as amplifying hosts of JEV; therefore, the domestic pig was considered to be a risk factor in the transmission of the disease to humans [2,3]. JEV is also an important pathogen in swine and causes considerable economic losses in pork production. The primary symptoms of pigs infected with JEV are fetal abortion and stillbirth in infected sows and aspermia in boars $[4,5]$. JEV has a single-stranded positive-sense RNA genome of approximately $11 \mathrm{~kb}$. The viral RNA encodes a single large polyprotein that is cleaved into three structural proteins,

\footnotetext{
*Correspondence: fanbin@mail.hzau.edu.cn; shzhao@mail.hzau.edu.cn Key Laboratory of Agricultural Animal Genetics, Breeding and Reproduction, Ministry of Education \& College of Animal Science and Technology, Huazhong Agricultural University, Wuhan 430070, China
}

capsid (C), precursor membrane (prM) and envelope (E); and seven non-structural (NS) proteins, NS1, NS2a, NS2b, NS3, NS4a, NS4b and NS5. The JEV E protein is the major structural protein exposed on the surface of the virus particle and mediates binding and fusion during virus entry $[6,7]$.

Viruses enter cells through binding cellular receptors. The interactions between the viruses and receptors are highly specific, determining which cell types and species can be infected. Additionally, the entrance of viruses into the host cells involves several endocytic pathways, including clathrin-mediated, caveolae-mediated, cholesterol-dependent endocytosis, macropinocytosis/phagocytosis and other mechanisms $[8,9]$. Clathrin-mediated endocytosis (CME) is the best characterized of the endocytic mechanisms, and most viruses utilize this type of endocytosis to enter cells. Recent studies have shown that JEV infects neuronal cells through a clathrin-independent, dynamin- and caveolae-mediated endocytosis pathway [10,11]. Previous studies have found that JEV enters Vero and Huh7 
cells through a clathrin-dependent pathway [12,13]. In addition, JEV internalisation into neural stem cells occurs by clathrin-mediated, caveolae independent endocytosis [14]. Persistent JEV infection has been demonstrated in porcine kidney cells [15] and numerous studies on JEV have been conducted in porcine kidney cells [16-20]. Moreover, vimentin has been identified as mediating the entry of JEV into porcine kidney cells [21]. However, the precise entry mechanism for JEV internalization into porcine cells remains unclear.

In this study, we define the role of cholesterol in JEV infection through cholesterol depletion, which significantly decreased JEV infection. In addition, we used RNA interference (RNAi) to examine the roles of clathrin and caveolin-1 in the JEV entry process; the results indicated that knockdown of clathrin reduced JEV infection, however, knockdown of caveolin-1 showed only a small effect on JEV infection and JEV entry was not affected by genistein. These results indicate that JEV endocytosis in PK15 cells is dependent on cholesterol and clathrin but not on caveolae.

\section{Results}

JEV infection is inhibited by the depletion of cholesterol

Many viruses commonly use lipid rafts to enter host cells. Cholesterol is a prominent component of lipid rafts. Membrane cholesterol can be disrupted by pharmacological agents, in which $\mathrm{M} \beta \mathrm{CD}$ extracts membrane cholesterol selectively [22], resulting in lipid raft disruption. Previous studies showed that the depletion of cholesterol could inhibit JEV infection during early stages $[11,14,23]$. To determine whether the removal of cholesterol affected the infection of PK15 cells with JEV, cells were treated with $10 \mathrm{nM} \mathrm{M \beta CD}$ and then incubated with JEV. After treatment, the internalization of JEV into cells was determined by immunofluorescence staining. As shown in Figure 1A, the treatment of the cells with $10 \mathrm{mM} M B C D$ significantly decreased JEV infection compared with the untreated control $(0 \mathrm{mM})$. In addition, after the cells were treated with 1,5 , or $10 \mathrm{mM}$ $\mathrm{M} \beta \mathrm{CD}$ and then infected with JEV, western blot analysis showed that the expression of JEV E protein was also
A

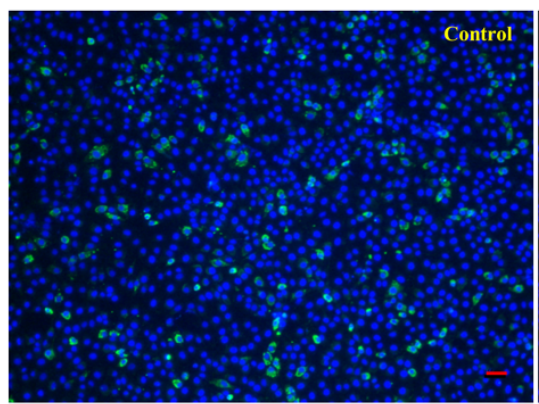

B

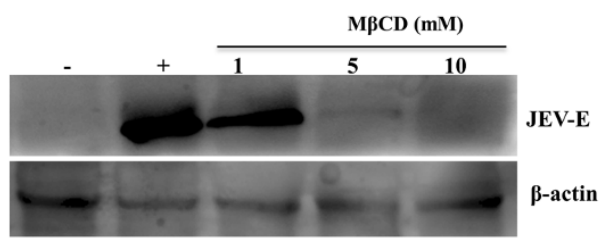

C

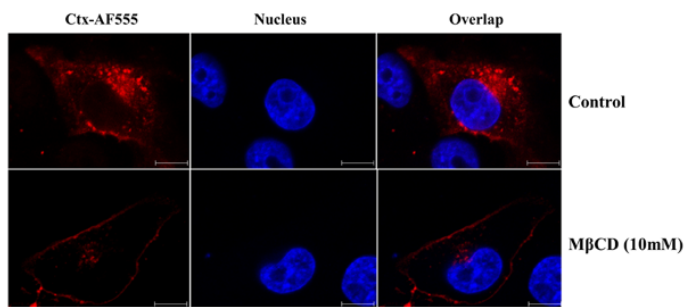

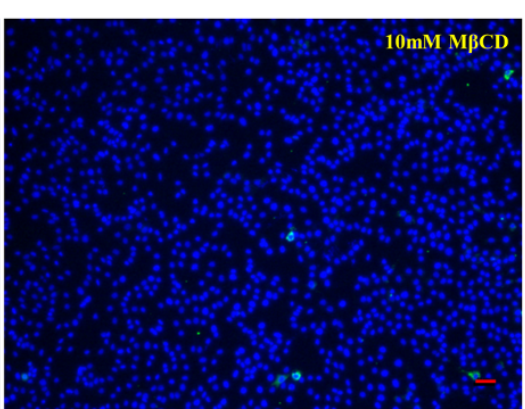

D

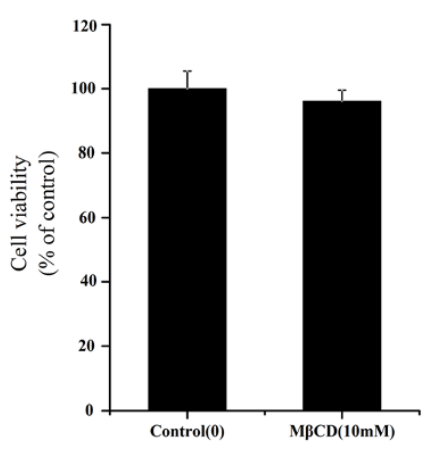

Figure 1 Depletion of cholesterol by M $\beta C D$ inhibits JEV infection. (A) PK15 cells were treated with $10 \mathrm{mM} M \beta C D$ for $1 \mathrm{~h}$ and infected with JEV in the presence of inhibitor; 36 hours post-infection (hpi), the cells were fixed and stained with anti-JEV E primary and Alexa488 anti-mouse IgG secondary antibodies. The nuclei were stained with DAPI. Scale bars, $50 \mu \mathrm{m}$. (B) The cells were treated with M $\beta C D$ as described above and then infected with JEV; $36 \mathrm{hpi}$, the cells were lysed and processed for western blot analysis of JEV E protein. $\beta$-actin was used as an internal loading control. +, control cells untreated with MBCD; -, control without JEV infection. (C) PK15 cells were untreated (control) or treated with 10 $\mathrm{mM} \mathrm{M} \beta C D$ for $1 \mathrm{~h}$ at $37^{\circ} \mathrm{C}$ and then incubated with $10 \mu \mathrm{g} / \mathrm{ml}$ AF 555-labelled Cholera toxin B (CTxB) for 30 min at $37^{\circ} \mathrm{C}$. Nuclei were stained with DAPI. Bar, $10 \mu \mathrm{m}$. (D) PK15 cells were plated on 96-well plates and then either left untreated (as a control) or treated with MBCD (10 mM) for $6 \mathrm{~h}$ at $37^{\circ} \mathrm{C}$. After treatment, cell viability was determined with a CCK-8 kit. The results are presented as the mean \pm SD of three independent experiments. 
inhibited. As shown in Figure 1B, JEV E protein expression was significantly inhibited by $\mathrm{M} \beta C D$ at the concentrations of $5 \mathrm{mM}$ or higher. These concentrations of $\mathrm{M} \beta C D$ were previously reported to significantly deplete membrane cholesterol $[11,24]$. Cholera toxin B (CTxB) is internalized in a lipid-raft-dependent manner after binding to its receptor GM1 ganglioside [25]. To confirm that $M \beta C D$ treatment resulted in inhibition of $\mathrm{CTxB}$, we analyzed the effect of the drug on $\mathrm{CTxB}$ uptake. As shown in Figure $1 \mathrm{C}$, untreated cells allowed CTxB uptake and showed a clear dotted perinuclear cytoplasmic fluorescence, whereas CTxB internalization was blocked when cells were treated with $M \beta C D$, confirming that lipid-raft-dependent endocytosis was inhibited. Cell proliferation and toxicological tests were determined by the CCK- 8 kit. The results showed that the cells treated with $10 \mathrm{mM} \mathrm{M} \beta \mathrm{CD}$ had no significant difference of absorbance from the control (Figure 1D). These observations suggested that JEV infection is dependent on the cellular membrane cholesterol content.

\section{Entry of JEV into PK15 cells is caveolae independent}

Recently, it has been shown that JEV enters rat neuroblastoma cells through a caveolae-mediated endocytosis pathway [11]. caveolin-1 is the principal component of caveolae. The knockdown of caveolin-1 by RNA interference (RNAi) decreased the number of membrane caveolae [26]. To test whether the entry of JEV into PK15 cells occurs via caveolae-mediated endocytosis, PK15 cells were transfected with siRNA targeting caveolin-1 or control siRNA and then infected with JEV. A small (although statistically significant) effect on JEV infection was observed between the caveolin-1 knockdown cells and cells expressing control siRNA (Figure 2A). Western blot results showed that the cells transfected with siCAV1 did not have a marked inhibition in JEV E protein expression (Figure 2B). The silencing efficiency of siCAV1 were analyzed at $48 \mathrm{~h}$ post-transfection by western blot (Figure 2C). These results suggested a possible role for caveolae in JEV infection. However, genistein, a tyrosine kinase inhibitor known to disrupt caveola related endocytosis [27], had no effect on JEV infection at concentrations of 50, 100 and $200 \mu \mathrm{M}$ (Figure 3A and B).

Cell viability tests were determined by the CCK- 8 kit, minimal cellular cytotoxicity was observed in cells treated by $200 \mu \mathrm{M}$ genistein compared with control (Figure $3 \mathrm{C}$ ). These concentrations of genistein were previously reported to significantly inhibit JEV infection in

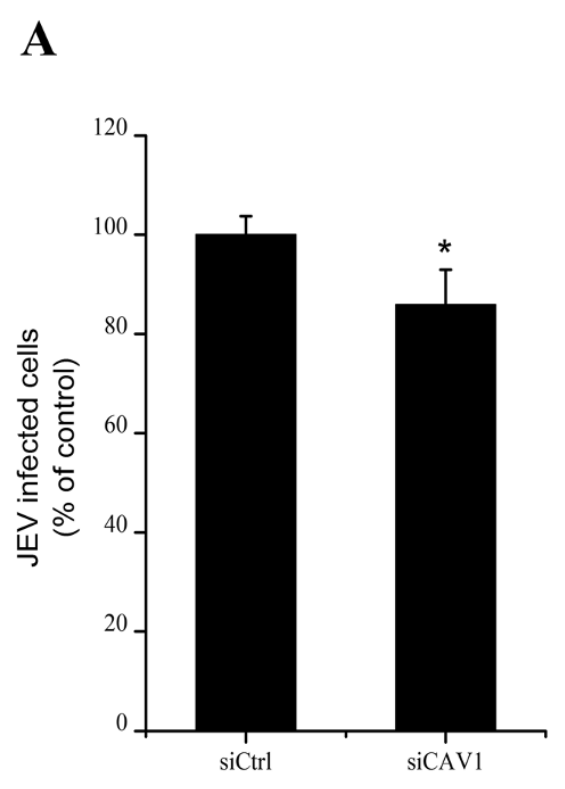

B
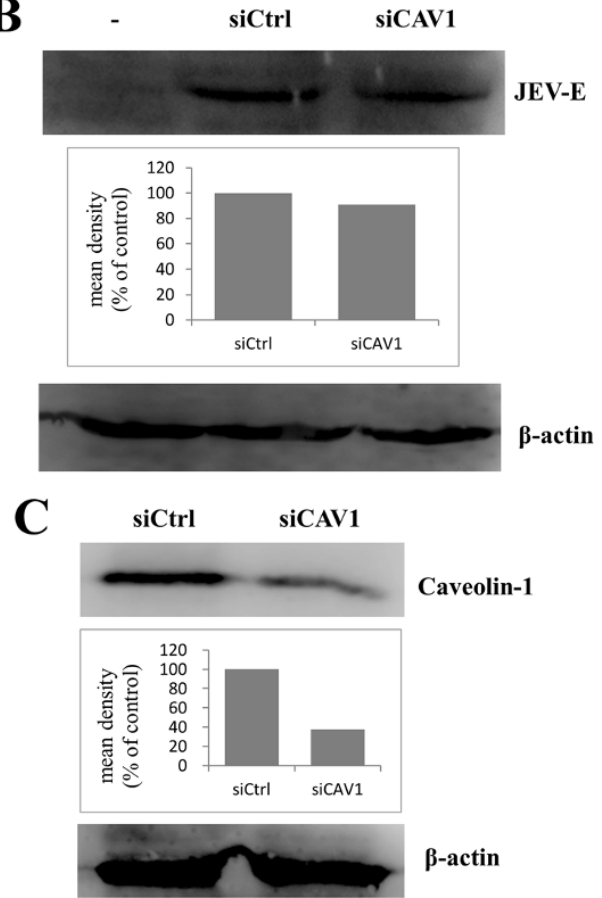

Figure 2 Caveolin-1 is not required for JEV entry. PK15 cells were transfected with siRNA targeting caveolin-1 or non-targeting siRNA (siCtrl) and infected with JEV at $48 \mathrm{~h}$ post-transfection, $36 \mathrm{hpi}$. (A) The percentage of internalized viruses of transfected cells was determined by flow cytometry and normalized to the value for the siCtrl. The results are presented as the mean \pm SD of three independent experiments. (B) JEV E protein was analyzed by western blot analysis. $\beta$-actin was used as an internal loading control. -, control without JEV infection. The mean densities of protein bands were measured by ImageJ software, and the results for each gel are shown as a bar graph under the immunoblot. (C) Caveolin-1 knockdown cells were analyzed at $48 \mathrm{~h}$ post-transfection by the immunoblotting. The mean densities of the protein bands are shown as a bar graph under the immunoblot. *, $p<0.05$. 


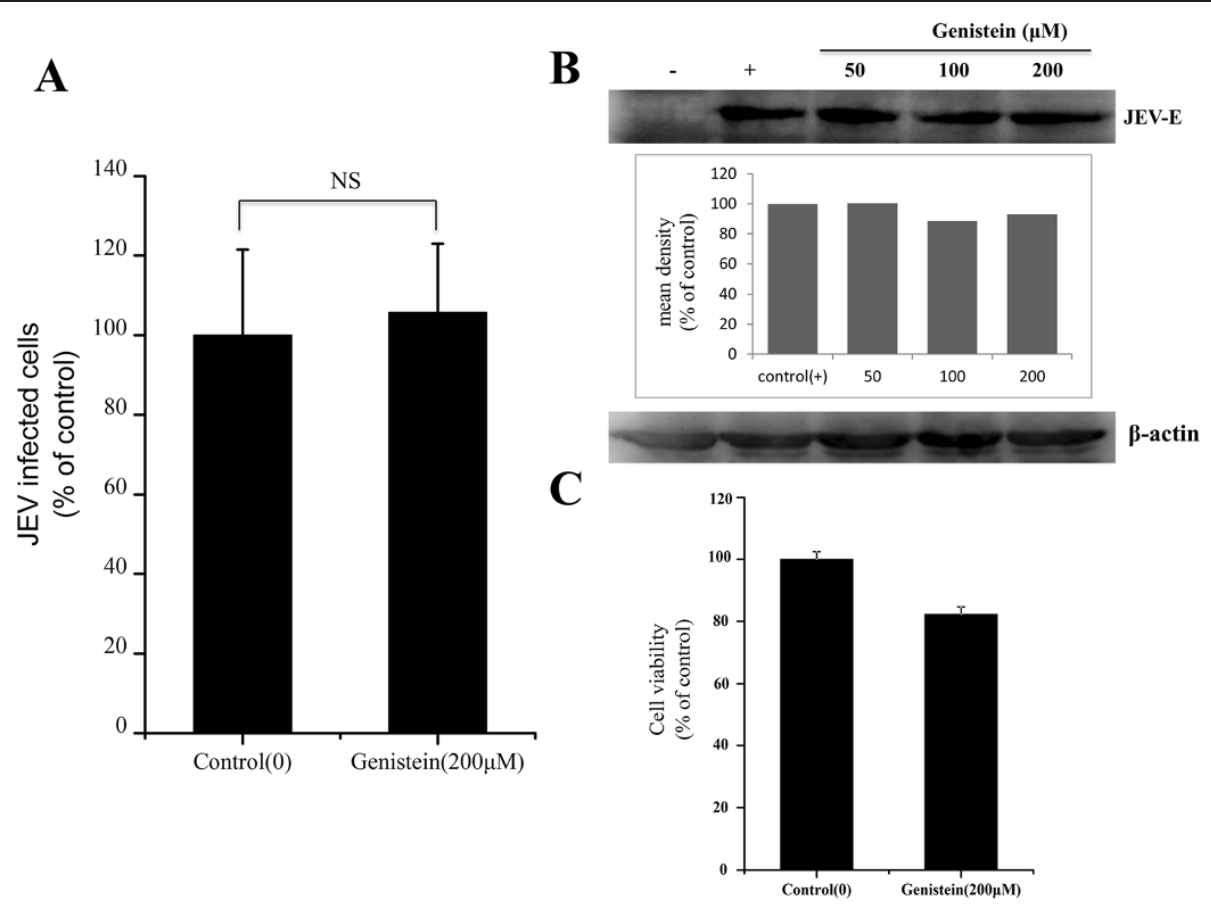

Figure 3 Effects of genistein on JEV infection. (A) PK15 cells were treated with $200 \mu \mathrm{M}$ genistein for $1 \mathrm{~h}$ and infected with JEV in the presence of inhibitor; 36 hours post-infection (hpi), the cells were fixed and stained with anti-JEV E primary and Alexa488 anti-mouse lgG secondary antibodies. The percentage of internalized viruses of drug-treated cells was determined by flow cytometry and normalized to the value for the control. NS, not significant. (B) The cells were treated with increasing concentrations of genistein as indicated, then infected with JEV; 36 hpi, the cells were lysed and processed for western blot analysis of JEV E protein. $\beta$-actin was used as an internal loading control. +, control cells untreated with genistein; -, control without JEV infection. The mean densities of the protein bands are shown as a bar graph under the immunoblot. (C) PK15 cells were untreated (control) or treated with genistein $(200 \mu \mathrm{M})$ for $6 \mathrm{~h}$ at $37^{\circ} \mathrm{C}$. Cell viability was determined by CCK-8 kit. The results are presented as the mean \pm SD.

rat neuroblastoma B104 cells [11]. These results suggest that, although caveolae-dependent processes may play some role in JEV infection, caveolae-mediated endocytosis is not the major route by which JEV enters the cell.

\section{JEV infects PK15 cells by clathrin-mediated endocytosis}

Early studies found that JEV entry into cells occurs by clathrin-mediated endocytosis and could be inhibited by chlorpromazine $[13,14]$. Thus, we examined whether clathrin-mediated endocytosis plays any role in JEV internalization. To specifically inhibit clathrin-mediated endocytosis, siRNAs were used to knockdown the expression of clathrin heavy chain $(\mathrm{CHC})$. PK15 cells were transfected with two independent specific siRNAs against $\mathrm{CHC}$ or a control siRNA and then infected with JEV. The CHC knockdown cells showed a significant reduction in JEV infection compared with cells transfected with control siRNA (Figure 4A). Western blot analysis showed that the expression of JEV E protein was also decreased in cells transfected with siCHC compared with control cells (Figure 4B). The silencing efficiency of siCHC-1 and siCHC-2 were analyzed at $48 \mathrm{~h}$ post-transfection by western blot (Figure 4C). These experiments suggest that JEV internalization in PK15 cells can occur via a clathrin-dependent mechanism.

\section{Discussion}

Lipid rafts are important for a number of cellular processes and play vital roles in virus entry [28]. Cholesterol is a prominent component of lipid rafts. Lipid rafts could be disrupted by depletion of cholesterol using chemical drugs, such as $M \beta C D$, nystatin and filipin [29-31], and some evidence suggests that cholesterol is involved in virus entry [24,32-34]. The inhibition of viral infection could be rescued by the addition of exogenous cholesterol. However, excess amounts of cholesterol block flaviviral infection [35]. Previous studies found that JEV could enter various cell types through a cholesteroldependent pathway $[11,13,14,23]$. In the present work, PK15 cells were used to investigate the role of membrane cholesterol in JEV entry. Similar results showed that membrane cholesterol was an absolute requirement for JEV infection. Cellular entry is initiated when flaviviral gpE binds to cell surface receptors [36], and JEV internalisation occurs in a lipid-raft dependent manner [14]. Therefore, the cellular receptors for JEV may located within the lipid raft domains. 


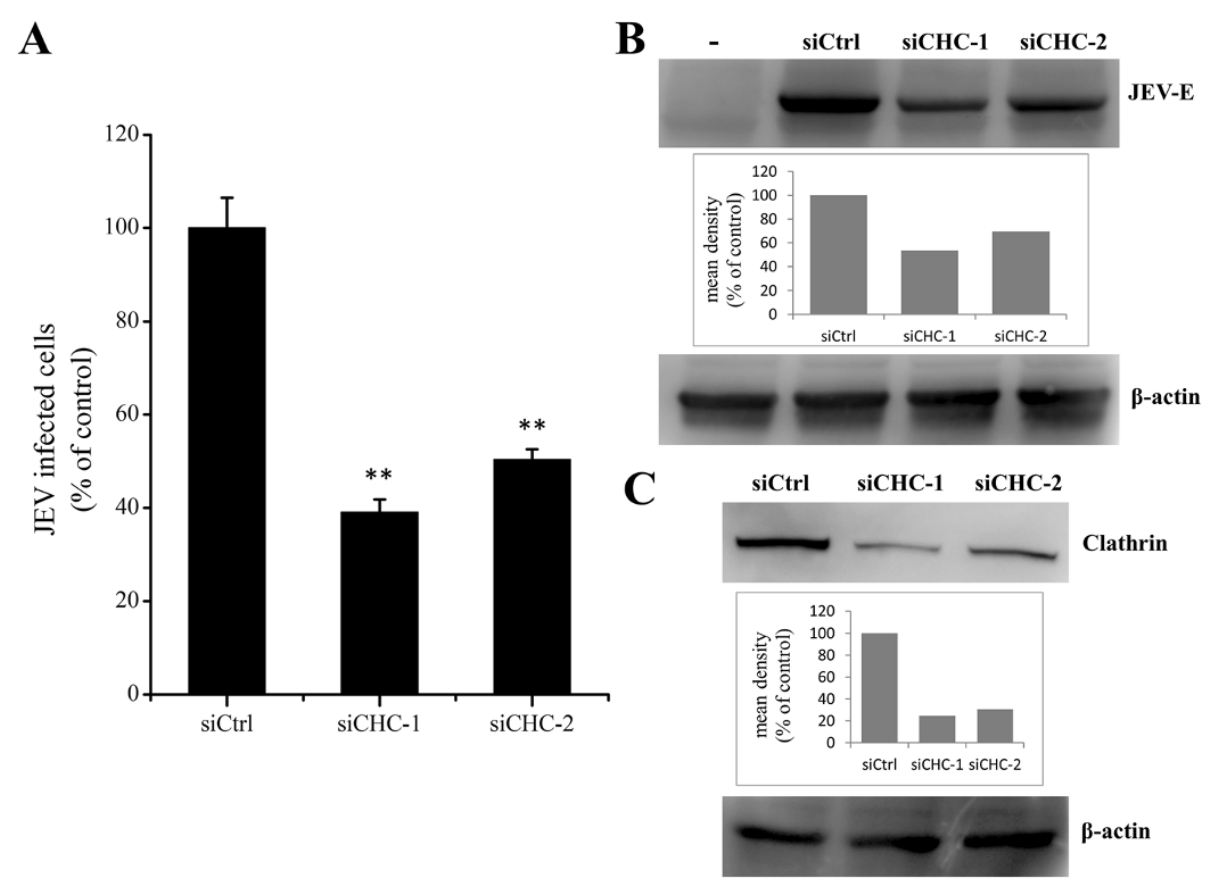

Figure 4 JEV entry depends on clathrin. PK15 cells were transfected with siRNA targeting clathrin heavy-chain (siCHC) or non-targeting siRNA (siCtrl) and infected with JEV at $48 \mathrm{~h}$ post-transfection. $36 \mathrm{hpi}$. (A) The percentage of internalized viruses of transfected cells was determined by flow cytometry and normalized to the value for the siCtrl. The results are presented as the mean \pm SD. (B) JEV E protein was analyzed by western blot analysis. $\beta$-actin was used as an internal loading control. -, control without JEV infection. The mean densities of the protein bands are shown as a bar graph under the immunoblot. (C) Clathrin knockdown cells were analyzed at $48 \mathrm{~h}$ post-transfection by the immunoblotting for clathrin. The mean densities of the protein bands are shown as a bar graph under the immunoblot. ${ }^{* *}, p<0.01$.

Viruses enter host cells through several endocytic pathways. Recent studies have found that JEV enters rat neuroblastoma cells via caveolae-mediated endocytosis [11]. Membrane cholesterol is required for caveolae formation, and caveolae-mediated endocytosis could be blocked by the depletion of cholesterol using $\mathrm{M} \beta \mathrm{CD}$ [37]. The present study shows that JEV entry is inhibited after cells were treated with $\mathrm{M} \beta C D$; thus, we speculated that caveolae was involved in JEV internalization. Therefore, drug treatment and small interfering RNA technology were used to validate whether JEV entry into PK15 cells was caveolae-dependent. Although the flow cytometry results showed that the cells transfected with caveolin-1 siRNA inhibited JEV infection by $15 \%$ (statistically significant), suggesting that caveolae-mediated pathway may play some role in the JEV entry into PK15 cells, JEV infection was not affected by genistein. We conclude that JEV entry into PK15 cells was caveolaeindependent. In addition, the receptor for JEV on PK15 cells is still uncharacterized. It is possible that a very small part of receptors was localized in caveolae, thus, the knockdown of caveolin-1 had a small effect on JEV infection.

Furthermore, $\mathrm{M} \beta \mathrm{CD}$, which destroys lipid raft structure by depleting cholesterol, also inhibits clathrincoated pit budding [38]. Therefore, we next examined the possibility of a clathrin-mediated pathway as an initial step in JEV entry into PK15 cells. Classic clathrinmediated endocytosis is commonly used in virus internalization, and JEV entry occurs via a classic clathrin-mediated pathway in neural stem cells and Vero cells $[13,14]$. In this study, JEV was also found to enter PK15 cells through the clathrin-dependent endocytic pathway. Based on published data and the results reported here, we assume that JEV is likely to infect different cell types by distinct pathways. The cellular receptors of JEV may be different in different cell types. However, a correlation between the cell receptors used and their entry pathway was unknown. Several potential cellular receptors of JEV were identified [12,39,40], but the genuine receptors of various cell types need to be identified to better understand the JEV pathogenic mechanism and to search for new therapeutic targets.

\section{Conclusion}

In the present study, we examined JEV infection using a chemical inhibitor and siRNA to disrupt different endocytic pathways in PK15 cells. Our results demonstrated that JEV entry was mediated by cholesterol- and clathrin-dependent, though not caveolin-1 dependent, pathways in PK15 cells. The mechanism of JEV entry in porcine cells was identified, and this research represents 
a new beginning for the development of new therapeutic targets in pigs.

\section{Materials and methods Cells and virus}

PK15 and baby hamster kidney (BHK-21) cell lines (obtained from the American Type Culture Collection, Manassas, VA) were cultured in Dulbecco's Modified Eagle Medium (DMEM, Thermo Scientific HyClone, Beijing, China) supplemented with $10 \%$ fetal bovine serum and maintained in a humidified incubator at $37^{\circ} \mathrm{C}$ and $5 \% \mathrm{CO}_{2}$. The JEV attenuated strain SA14-14-2 (GenBank accession: AF315119.1) was used in this study. Virus was propagated in BHK-21 cells. To generate virus stocks, BHK-21 cells were grown in monolayers of 75$\mathrm{cm}^{2}$ flasks, and the cells were infected with JEV until $90 \%$ confluence was reached. The cells were harvested when the cytopathic effect was extensive (48 h). Virions were collected through three freeze-thaw cycles and centrifugation. Virus titers were determined by the $50 \%$ tissue culture infectious dose (TCID50) [41], and the virus suspensions at a multiplicity of infection (MOI) of 1 were utilized for all infection experiments.

\section{Antibodies and reagents}

Mouse anti-JEV E antibody was used for immunofluorescence assays and western blot analysis. Polyclonal rabbit anti $\beta$-actin, caveolin-1 and clathrin heavy chain antibodies were purchased from Cell Signaling Technology, Inc. (3 Trash Lane, Danvers, MA 01923, US). The Cell Counting Kit 8 (CCK-8), DAPI and genistein were purchased from Beyotime Biotechnology, Inc. (Jiangsu, China). Alexa Fluor 488-labeled goat antimouse IgG and Alexa Fluor 555-conjugated Cholera toxin $\mathrm{B}(\mathrm{CTxB})$ were purchased from Molecular Probes (Eugene, OR). HRP-conjugated goat-anti-rabbit and goat-anti-mouse secondary antibodies were purchased from Proteintech Group, Inc (Wuhan, China). Methyl$\beta$-cyclodextrin $(\mathrm{M} \beta \mathrm{CD})$ was purchased from SigmaAldrich (St. Louis, MO) and dissolved in water.

\section{Drug treatments and virus infection}

PK15 cells were plated on 6-well plates and grown to approximately $80 \%$ confluence at $37^{\circ} \mathrm{C}$ in a $\mathrm{CO}_{2}$ incubator. The cells were washed three times with phosphatebuffered saline (PBS) and then pre-treated with $\mathrm{M} \beta C D$ or genistein for $1 \mathrm{~h}$ at $37^{\circ} \mathrm{C}$ at various concentrations. After treatment, the cells were infected with JEV for $1 \mathrm{~h}$ at $37^{\circ} \mathrm{C}$ in the presence of the chemical. The cells were washed to remove excess virus and drug, then further incubated in cell culture medium containing 2\% FBS at $37^{\circ} \mathrm{C}$ with $5 \% \mathrm{CO}_{2}$. Western blot and immunofluorescence staining analysis for JEV E protein expression were performed $36 \mathrm{~h}$ post-infection.

\section{RNA interference experiments}

For small interfering RNA (siRNA) analysis, siRNA oligonucleotides with specificity for porcine CAV1 and clathrin heavy chain $(\mathrm{CHC})$ and non-targeting siRNA (siCtrl) were synthesized from GenePharma (Shanghai, China). The target sequence of siCAV1 was as follow: siCAV1-1 5'- CACACAGUUUCGAUGGCAUCUTT-3'. Sequences of siCHC-1 (equal mixture of the two RNA duplex oligonucleotides) were as follows: 5'-AAGC UGGGAAAACUCUUCAGA-3', 5'-UAAUCCAAUUCG AAGACCAAU-3' [42], siCHC-2: 5'-GGCCCAGGUG GUAAUUAUUUU-3'. PK15 cells were transfected with 50 nM siRNA using Lipofectamine 2000 (Invitrogen) according to the manufacturer's instructions. Subsequent experiments were performed $48 \mathrm{~h}$ post-transfection. Knockdown efficiencies were quantified by western blot analysis.

\section{Western blot analysis}

Cells were washed with PBS three times and lysed in a modified radioimmunoprecipitation assay (RIPA) lysis buffer (50 mM Tris (pH 7.4), $150 \mathrm{mM} \mathrm{NaCl,} \mathrm{1 \%} \mathrm{Triton}$ $\mathrm{X}-100,1 \%$ sodium deoxycholate, $0.1 \%$ SDS, $1 \mathrm{mM}$ phenylmethylsulfonyl fluoride [PMSF]). Protein concentrations were determined with a BCA Protein Assay kit (Solarbio, China). An equal amount of protein lysate was separated by $12 \%$ SDS-polyacrylamide gels and transferred to PVDF membranes (Millipore, Bedford, MA). The membranes were blocked with 5\% nonfat milk in tris-buffered saline containing $0.1 \%$ tween-20 (TBST) and then incubated with primary antibodies overnight at $4^{\circ} \mathrm{C}$. The membranes were washed three times with TBST (each wash was $10 \mathrm{~min}$ ), and HRP-conjugated goat anti-rabbit or goat-anti-mouse secondary antibody (1:3000 dilutions in blocking buffer) was added. Bound antibodies were visualized by SuperSignal West Pico chemiluminescent substrate (Pierce, Rockford, IL). The mean densities of protein bands were measured by ImageJ software (National Institutes of Health, Bethesda, Maryland).

\section{Immunofluorescence assays}

The cells were fixed with $4 \%$ paraformaldehyde for 20 min at room temperature (RT) and permeabilized with $0.2 \%$ Triton X-100 for $15 \mathrm{~min}$. The cells were then incubated with a blocking buffer (PBS containing 5\% bovine serum albumin [BSA]) at $37^{\circ} \mathrm{C}$ for $30 \mathrm{~min}$. After three washes with PBS, the cells were stained with anti-JEV E mouse antibody at room temperature for $1 \mathrm{~h}$. After being washed with PBS, the cells were incubated with Alexa Fluor 488 conjugated goat anti-mouse antibody (1:200 dilutions in blocking buffer) IgG. The nuclei were stained with DAPI. 


\section{Flow cytometry}

PK15 cells infected with JEV were washed one time with PBS, detached and transferred to $1.5 \mathrm{ml}$ centrifuge tubes. The cells were centrifuged at $1000 \mathrm{rpm} / \mathrm{min}$ for $10 \mathrm{~min}$ and fixed with $4 \%$ paraformaldehyde for $15 \mathrm{~min}$ at room temperature. After being permeabilized with Triton $\mathrm{X}-100$, the cells were incubated with anti-JEV E mouse antibody overnight at $4^{\circ} \mathrm{C}$. The cells were washed 3 times with PBS then incubated with Alexa Fluor 488 goat anti-mouse IgG at 1:200 for $1 \mathrm{~h}$ at room temperature. The cells were washed with PBS, resuspended in $500 \mu \mathrm{l}$ PBS and analyzed using FACScan flow cytometer with CellQuest pro software (BD Biosciences, San Jose, CA). The cells were counted as infected if their fluorescence densities were greater than the intensity of the uninfected cells. The amount of infected cells relative to the untreated or siCtrltransfected controls was given as percent infection. At least 10,000 cells were analyzed per sample.

\section{Statistical analysis}

The results were presented as the mean \pm standard deviation (SD). Statistical significance was assessed by Student's t-test, and statistical significance was ascribed when $p<0.05$.

\section{Competing interests}

The authors declare that they have no competing interests.

\section{Authors' contributions}

SBY carried out most of the experiments and drafted the manuscript. MHH, $\mathrm{XYL}$ and $\mathrm{XDL}$ participated in part of experiments. BF and $\mathrm{SHZ}$ designed the study, supervised the work and edited the final version of this manuscript. All authors have read and approved the final version of the manuscript.

\section{Acknowledgments \\ We thank Professor Shengbo Cao (Huazhong Agriculture University, Wuhan China) for generously providing the mouse anti-JEV E antibody and JEV attenuated strain SA14-14-2. This work was supported by the National High Technology Plan (863, 2013AA102502), the National Science Foundation of China $(31101693,31072009)$, the Fundamental Research Funds for the Central Universities (2010PY008).}

Received: 27 April 2013 Accepted: 9 August 2013

Published: 12 August 2013

\section{References}

1. Misra UK, Kalita J: Overview: Japanese encephalitis. Prog Neurobiol 2010, 91:108-120.

2. Solomon T: Control of Japanese encephalitis-within our grasp? N Engl J Med 2006, 355:869-871.

3. Erlanger TE, Weiss S, Keiser J, Utzinger J, Wiedenmayer K: Past, present, and future of Japanese encephalitis. Emerg Infect Dis 2009, 15:1-7.

4. Takashima I, Watanabe T, Ouchi N, Hashimoto N: Ecological studies of Japanese encephalitis virus in Hokkaido: interepidemic outbreaks of swine abortion and evidence for the virus to overwinter locally. Am J Trop Med Hyg 1988, 38:420-427.

5. Burns KF: Congenital Japanese $B$ encephalitis infection of swine. Proc Soc Exp Biol Med 1950, 75:621-625.

6. McMinn PC: The molecular basis of virulence of the encephalitogenic flaviviruses. J Gen Virol 1997, 78(Pt 11):2711-2722.
7. Allison SL, Schalich J, Stiasny K, Mandl CW, Heinz FX: Mutational evidence for an internal fusion peptide in flavivirus envelope protein E. $J$ Virol 2001, 75:4268-4275.

8. Marsh M, Helenius A: Virus entry: open sesame. Cell 2006, 124:729-740.

9. Mercer J, Schelhaas M, Helenius A: Virus entry by endocytosis. Annu Rev Biochem 2010, 79:803-833.

10. Kalia M, Khasa R, Sharma M, Nain M, Vrati S: Japanese encephalitis virus infects neuronal cells through a clathrin-independent endocytic mechanism. J Virol 2013, 87:148-162.

11. Zhu YZ, Xu QQ, Wu DG, Ren H, Zhao P, Lao WG, Wang Y, Tao QY, Qian XJ, Wei YH, Cao MM, Qi ZT: Japanese encephalitis virus enters rat neuroblastoma cells via a pH-dependent, dynamin and caveolamediated endocytosis pathway. J Virol 2012, 86:13407-13422.

12. Tani H, Shiokawa M, Kaname Y, Kambara H, Mori Y, Abe T, Moriishi K, Matsuura Y: Involvement of ceramide in the propagation of Japanese encephalitis virus. J Virol 2010, 84:2798-2807.

13. Nawa M, Takasaki T, Yamada K, Kurane I, Akatsuka T: Interference in Japanese encephalitis virus infection of Vero cells by a cationic amphiphilic drug, chlorpromazine. J Gen Virol 2003, 84:1737-1741.

14. Das S, Chakraborty S, Basu A: Critical role of lipid rafts in virus entry and activation of phosphoinositide 3 ' kinase/Akt signaling during early stages of Japanese encephalitis virus infection in neural stem/progenitor cells. J Neurochem 2010, 115:537-549.

15. Shah PS, Gadkari DA: Persistent infection of porcine kidney cells with Japanese encephalitis virus. Indian J Med Res 1987, 85:481-491.

16. Espada-Murao LA, Morita K: Delayed cytosolic exposure of Japanese encephalitis virus double-stranded RNA impedes interferon activation and enhances viral dissemination in porcine cells. J Virol 2011, 85:6736-6749.

17. Verma SK, Gupta N, Pattnaik P, Babu JP, Rao PV, Kumar S: Antibodies against refolded recombinant envelope protein (domain III) of Japanese encephalitis virus inhibit the JEV infection to Porcine Stable Kidney cells. Protein Pept Lett 2009, 16:1334-1341.

18. Lad VJ, Gupta AK: Inhibition of Japanese encephalitis virus maturation and transport in PS cells to cell surface by brefeldin A. Acta Virol 2002 46:187-190

19. Lad VJ, Shende VR, Gupta AK, Koshy AA, Roy A: Effect of tunicamycin on expression of epitopes on Japanese encephalitis virus glycoprotein $E$ in porcine kidney cells. Acta Virol 2000, 44:359-364.

20. Mori Y, Yamashita T, Tanaka Y, Tsuda Y, Abe T, Moriishi K, Matsuura Y: Processing of capsid protein by cathepsin $L$ plays a crucial role in replication of Japanese encephalitis virus in neural and macrophage cells. J Virol 2007, 81:8477-8487.

21. Das S, Ravi V, Desai A: Japanese encephalitis virus interacts with vimentin to facilitate its entry into porcine kidney cell line. Virus Res 2011, 160:404-408

22. Ilangumaran S, Hoessli DC: Effects of cholesterol depletion by cyclodextrin on the sphingolipid microdomains of the plasma membrane. Biochem J 1998, 335(Pt 2):433-440.

23. Kalia M, Khasa R, Sharma M, Nain M, Vrati S: Japanese Encephalitis Virus Infects Neuronal Cells through a Clathrin Independent Endocytic Mechanism. J Virol 2012, 87:148-162.

24. Guo CJ, Liu D, Wu YY, Yang XB, Yang LS, Mi S, Huang YX, Luo YW, Jia KT, Liu $Z Y$, Chen WJ, Weng SP, Yu XQ, He JG: Entry of tiger frog virus (an Iridovirus) into HepG2 cells via a pH-dependent, atypical, caveolamediated endocytosis pathway. J Virol 2011, 85:6416-6426.

25. Fujinaga Y, Wolf AA, Rodighiero C, Wheeler H, Tsai B, Allen L, Jobling MG, Rapoport T, Holmes RK, Lencer WI: Gangliosides that associate with lipid rafts mediate transport of cholera and related toxins from the plasma membrane to endoplasmic reticulm. Mol Biol Cell 2003, 14:4783-4793.

26. Gonzalez-Munoz E, Lopez-Iglesias C, Calvo M, Palacin M, Zorzano A, Camps M: Caveolin-1 Loss of Function Accelerates Glucose Transporter 4 and Insulin Receptor Degradation in 3T3-L1 Adipocytes. Endocrinology 2009, 150:3493-3502.

27. Querbes W, Benmerah A, Tosoni D, Di-Fiore PP, Atwood WJ: A JC virusinduced signal is required for infection of glial cells by a clathrin- and eps15-dependent pathway. J Virol 2004, 78:250-256.

28. Manes S, Del-Real G, Martinez AC: Pathogens: raft hijackers. Nat Rev Immunol 2003, 3:557-568.

29. Neufeld EB, Cooney AM, Pitha J, Dawidowicz EA, Dwyer NK, Pentchev PG, Blanchette-Mackie EJ: Intracellular trafficking of cholesterol monitored with a cyclodextrin. J Biol Chem 1996, 271:21604-21613. 
30. Orlandi PA, Fishman PH: Filipin-dependent inhibition of cholera toxin: evidence for toxin internalization and activation through caveolae-like domains. J Cell Biol 1998, 141:905-915.

31. Rothberg KG, Heuser JE, Donzell WC, Ying YS, Glenney JR, Anderson RG: Caveolin, a protein component of caveolae membrane coats. Cell 1992, 68:673-682.

32. Perry JW, Wobus CE: Endocytosis of murine norovirus 1 into murine macrophages is dependent on dynamin II and cholesterol. J Virol 2010, 84:6163-6176.

33. Vela EM, Zhang L, Colpitts TM, Davey RA, Aronson JF: Arenavirus entry occurs through a cholesterol-dependent, non-caveolar, clathrinmediated endocytic mechanism. Virology 2007, 369:1-11.

34. Huang L, Zhang YP, Yu YL, Sun MX, Li C, Chen PY, Mao X: Role of lipid rafts in porcine reproductive and respiratory syndrome virus infection in MARC-145 cells. Biochem Biophys Res Commun 2011, 414:545-550.

35. Lee CJ, Lin HR, Liao CL, Lin YL: Cholesterol effectively blocks entry of flavivirus. J Virol 2008, 82:6470-6480.

36. Perera R, Khaliq M, Kuhn RJ: Closing the door on flaviviruses: entry as a target for antiviral drug design. Antiviral Res 2008, 80:11-22.

37. Rodal SK, Skretting G, Garred O, Vilhardt F, van-Deurs B, Sandvig K: Extraction of cholesterol with methyl-beta-cyclodextrin perturbs formation of clathrin-coated endocytic vesicles. Mol Biol Cell 1999, 10:961-974

38. Subtil A, Gaidarov I, Kobylarz K, Lampson MA, Keen JH, McGraw TE: Acute cholesterol depletion inhibits clathrin-coated pit budding. Proc Natl Acad Sci U S A 1999, 96:6775-6780.

39. Zhu YZ, Cao MM, Wang WB, Wang W, Ren H, Zhao P, Qi ZT: Association of heat-shock protein 70 with lipid rafts is required for Japanese encephalitis virus infection in Huh7 cells. J Gen Virol 2012, 93:61-71.

40. Ren J, Ding T, Zhang W, Song J, Ma W: Does Japanese encephalitis virus share the same cellular receptor with other mosquito-borne flaviviruses on the C6/36 mosquito cells? Virol J 2007, 4:83.

41. Reed $L, H$ M: A simple method of estimating fifty percent endpoints. Am J Hygiene 1938, 27:493-497.

42. Motley A, Bright NA, Seaman MN, Robinson MS: Clathrin-mediated endocytosis in AP-2-depleted cells. J Cell Biol 2003, 162:909-918.

Cite this article as: Yang et al:: Japanese encephalitis virus infects porcine kidney epithelial PK15 cells via clathrin- and cholesteroldependent endocytosis. Virology Journal 2013 10:258.

\section{Submit your next manuscript to BioMed Central and take full advantage of:}

- Convenient online submission

- Thorough peer review

- No space constraints or color figure charges

- Immediate publication on acceptance

- Inclusion in PubMed, CAS, Scopus and Google Scholar

- Research which is freely available for redistribution 